175

\section{Burning Mouth Syndrome Equivalent to Restless} Leg Syndrome

Priya Batta'; Sarosh Majeed' ; Davinder Dhillon'; and Alan R Hirsch ${ }^{2}$

${ }^{1}$ Windsor University School of Medicine, Basseterre, St. Kitts

${ }^{2}$ Smell and Taste Treatment and Research Foundation, Chicago, IL

ABSTRACT: Study Objective: Stuginski-Barbosa reported a patient with Burning Mouth Syndrome (BMS), whose symptoms were transiently improved with tongue movement, postulated to be an oral equivalent of restless leg syndrome (RLS) (Stuginski-Barbosa, 2008).

METHODS: Four Case Studies are described.

RESULTS: Case 1: A 35 year old pathological left handed woman presented with one-year history of burning and stinging sensations on the sides of the tongue and lips without any known precipitant. It is reduced with chewing gum and eating. For the last few months she noticed tongue movement reduced the burning sensation. Two to three seconds of tongue movement alleviated the burning from $3 / 10$ to $0 / 10$ in intensity, which persists for one minute after cessation of tongue movement.

Case 2: A 57 year old right handed female, three years prior to presentation, had multiple teeth extractions. Four months later, she perceived a rough sensation of her tongue, which has gradually changed to a burning sensation which has persisted since then. The burning is $10 / 10$ in severity and constant. Chewing 24 pieces of gum a day reduces the pain. Movement of the tongue including anterior-posterior protrusion and retraction, side to side movements without contacting surrounding oral mucosa, converted pain from $9 / 10$ to $5 / 10$, which lasted approximately 1 minute.

Case 3: Eight years prior to presentation, this 60 year old woman developed BMS, after dental extraction. The pain involves the tongue (right and center of palate) accompanied by a gritty sandpaper-like sensation. The burning is a level 5/10 in intensity and progressively worsens throughout the day. Burning mouth pain is made less intense with chewing gum and using dental wax. Baseline burning mouth severity is at level 5/10 however, with protrusion, retraction, and side-to-side movement of the tongue, the intensity dropped to $3 / 10$.

Case 4: Three years prior to presentation, a 59 year old female patient suffered severe allergies with onset of burning mouth and absent taste. The burning is in the middle of her tongue, rarely, her palate, but never on her lips. Severity is $2 / 10$ and is worse in the morning, with drinking red wine, and hot drinks. It is better with cold drinks and ice cream. Movement of her tongue side-to-side eliminates the burning severity from $2 / 10$ to $0 / 10$, lasting one minute.

CONCLUSION: Like the patients described by Stuginski \& Barbosa 2008, these four patients showed reduced pain in response to tongue kinesia (Stuginski-Barbosa, 2008). Their mandibulation of gum as an analgesic maneuver may have been due, not to the taste of the gum, but rather to the associated tongue movement that mastication induces. Through moving, large nerve fibers of proprioception are stimulated, which may act through the Gate Controlled Theory of Pain of Melzac and Wall, to reduce unrestricted small nerve fiber discharge, thus inhibiting pain. Query regarding movement or gum chewing inhibition of BMS is warranted.

FUNDING ACKNOWLEDGEMENTS: Smell \& Taste Treatment and Research Foundation.

\section{6}

\section{The Influence of Chronic Stress on the} Development of Psychiatric Conditions

Pwint Phyu, MD'; Rhaisa Dumenigo, $M D^{2}$; Juan D. Oms, $\mathrm{MD}^{3}$; Luxhman Gunaseelan, MS-III ${ }^{4}$; Golbon Foroughi, MS-IV ; and Syed A. A. Rizvi, PhD

${ }^{1}$ Post Graduate Year 3, Psychiatry Department, Larkin Community Hospital, Miami, FL

${ }^{2}$ Attending Physician and Medical Director, Psychiatry Department, Larkin Community Hospital, Miami, FL

${ }^{3}$ Program Director, Department of Psychiatry, Larkin Community Hospital, Miami, FL

${ }^{4}$ Saba University School of Medicine, Saba, Caribbean Netherlands

${ }^{5}$ Professor, Department of Pharmaceutical Sciences, College of Pharmacy, Nova Southeastern University,

Fort Lauderdale, FL

ABSTRACT: A 20-year-old woman with a history of bipolar disorder (BD), posttraumatic stress disorder (PTSD), and borderline personality disorder (BPD) presented to the emergency department with suicidal ideation. This report compares her clinical course with that of her twin brother, who also had received a BD diagnosis but grew up in a less stressful environment than his sister. It also describes the possible genetic and environmental etiologic factors associated with $\mathrm{BD}$, PTSD, BPD, and other psychiatric conditions. Chronic stress is strongly associated with the development of these conditions; pathologic processes involving the effect of stress hormones and neurotransmitters on the brain and central nervous system are described, along with pharmacologic and nonpharmacologic approaches to reducing chronic stress in persons with BD and other psychological disorders.

FUNDING ACKNOWLEDGEMENTS: No funding. 